\title{
Criminologie
}

\section{Enfance et détention correctionnelle. Le cas du tribunal des jeunes de Winnipeg entre 1930 et 1959}

\section{Bruno Théorêt}

Volume 28, numéro 1, 1995

L'histoire de l'enfermement et des populations pénales

URI : https://id.erudit.org/iderudit/017367ar

DOI : https://doi.org/10.7202/017367ar

Aller au sommaire du numéro

\section{Éditeur(s)}

Les Presses de l'Université de Montréal

ISSN

0316-0041 (imprimé)

1492-1367 (numérique)

Découvrir la revue

Citer cet article

Théorêt, B. (1995). Enfance et détention correctionnelle. Le cas du tribunal des jeunes de Winnipeg entre 1930 et 1959. Criminologie, 28(1), 119-138.

https://doi.org/10.7202/017367ar
Résumé de l'article

In 1908, the first Canadian juvenile court was established in Winnipeg. The study of the functioning of this court during the period 1930-1959 shows that the judicial process and the sentencing decisions were in relation with two different approaches to delinquency. The first approach is the criminal one. Conceptually, this approach is close to the justice model, borrowed from adult courts which operate with retribution and deterrence. The second approach is the socio-penal approach. This approach is linked to the legal (and social) status of children characterized by the obligations of acceptable adult supervision, fixed place of residence, restrained presence in public places and sexual moralization. It includes types of delinquencies for which adults cannot be incriminated and delinquencies related to contacts with the Court. The sentencing of girls and boys whose files correspond to the criminal model is characterized by the recourse to fining, reprimand, probation and restitution while those whose files match the socio-penal model are punished by correctional detention, probation, release and fine. 


\section{ENFANCE ET DÉTENTION CORRECTIONNELLE. LE CAS DU TRIBUNAL DES JEUNES DE WINNIPEG \\ ENTRE 1930 ET 1959 Bruno Théorêt ${ }^{1}$}

In 1908, the first Canadian juvenile court was established in Winnipeg. The study of the functioning of this court during the period 1930-1959 shows that the judicial process and the sentencing decisions were in relation with two different approaches to delinquency. The first approach is the criminal one. Conceptually, this approach is close to the justice model, borrowed from adult courts which operate with retribution and deterrence. The second approach is the socio-penal approach. This approach is linked to the legal (and social) status of children characterized by the obligations of acceptable adult supervision, fixed place of residence, restrained presence in public places and sexual moralization. It includes types of delinquencies for which adults cannot be incriminated and delinquencies related to contacts with the Court. The sentencing of girls and boys whose files correspond to the criminal model is characterized by the recourse to fining, reprimand, probation and restitution while those whose files match the socio-penal model are punished by correctional detention, probation, release and fine.

\section{INTRODUCTION}

Dans cet article, nous examinons l'imposition de sentences de détention correctionnelle par le tribunal des jeunes de Winnipeg entre 1930 et 1959, en opposant ce type de sentences aux autres sentences imposées par le tribunal. Nous allons montrer que, pour une partie de la population de jeunes qui se sont présentés devant le tribunal, les conditions de vie et de contrôle de même que le type de délinquance dont ils ont été reconnus coupables permettent de comprendre la détention correctionnelle qui leur est imposée. Nous allons aussi montrer qu'à cet égard, le fonctionnement du tribunal des jeunes est en conformité avec le droit des jeunes qui le régit. Nous proposons d'aborder ces questions dans un cadre socio-historique spécifique, celui du tribunal des jeunes de Winnipeg durant les années 1930-1959. Notre examen porte sur le rapport entre les mesures sentencielles

1. Agent de recherche, Centre international de criminologie comparée (C.I.C.C.) et Groupe de recherche et d'analyse sur les politiques et les pratiques pénales (G.R.A.P.P.P.), Université de Montréal. L'auteur désire remercier Mme Danielle Laberge, M. Len Kaminski et M. Jean Trépanier pour leurs précieux conseils dans l'analyse et la rédaction de cet article. 
imposées aux jeunes et ce que nous appelons les approches criminelle et socio-pénale de fonctionnement du tribunal. Ces approches, liées à des situations-types, nous semblent mieux représenter les réalités juridique et pratique du fonctionnement des tribunaux et être mieux à même d'appréhender les mesures sentencielles qui sont imposées.

À cet égard, il nous semble que le tribunal des jeunes de Winnipeg est un lieu particulièrement propice à cet examen. Premier tribunal des jeunes établi au Canada en vertu de la Loi sur les jeunes délinquants, il manifeste rapidement les caractéristiques modernes des institutions judiciaires canadiennes réservées aux jeunes. Les dossiers judiciaires de ce tribunal pour la période 1930-1950 ayant été conservés, leur dépouillement permet un tel examen.

Trois objectifs spécifiques sont poursuivis ici: premièrement, nous entendons montrer que le tribunal des jeunes de Winnipeg, opérant sous le régime de la Loi sur les jeunes délinquants de 1929, fonctionne selon deux approches liées à l'évaluation des conditions de vie et de contrôle des jeunes et à la nature de la délinquance des jeunes; deuxièmement, nous voulons confronter ces approches à des pratiques judiciaires de ce tribunal, tout particulièrement ses décisions sentencielles; enfin, troisièmement, nous entreprenons de montrer qu'à tout le moins en ce qui a trait à la nature et à la distribution des sentences imposées au groupe de jeunes dont les dossiers correspondent aux approches criminelle et socio-pénale, ce tribunal ne fait pas montre de pratiques sentencielles discriminatoires sur la base du sexe.

\section{Jeunes et enfermement}

Les jeunes Manitobains entre 1924 et 1959: ils doivent être sous le contrôle et en compagnie d'adultes responsables; ils doivent avoir un domicile fixe; ils doivent respecter un couvre-feu; ils doivent être à l'école durant le jour; ils ne doivent pas se trouver dans les lieux publics ni les débits d'alcool; ils ne doivent pas travailler à certaines heures du jour et de la nuit; ils doivent avoir une conduite morale en tous temps; enfin, ils doivent s'abstenir de contrevenir aux dispositions du droit crimine ${ }^{2}$. Alors, peut-être pourrions-nous être cynique en abordant la question de l'enfermement pénal des jeunes en posant la question suivante : Est-ce que l'enfermement pénal des jeunes est une suite logique de l'enfermement social dont ils sont l'objet au Manitoba depuis le début du $\mathrm{XX}^{\mathrm{e}}$ siècle?

2. Statut du Manitoba, 1924, C.6. An Act to Amend An Act Respecting the Welfare of Children. Une première version de cette loi fut adoptée en 1922. Après quelques modifications mineures en 1924, elle fut appliquée sur l'ensemble du territoire manitobain. 
L'enfermement des mineurs pénaux dont nous parlons renvoie tant à la représentation idéelle d'une décision prise dans le cadre d'une affaire judiciaire qu'à un ensemble de pratiques rationnelles caractérisées par le lieu où elles ont cours et la nature non-volontaire de la situation des objets de ces pratiques. La décision d'imposer l'enfermement est certes prise par un juge, mais très souvent en collaboration avec plusieurs intervenants dans le dossier d'un jeune. Selon que l'intervenant est juge, agent de probation, psychiatre ou même médecin, directeur d'établissement correctionnel ou responsable de sociétés d'aide à l'enfance, les représentations de l'effet attendu de cet enfermement sont multiples et peuvent aussi bien diverger que converger. On attend toutefois du lieu de l'enfermement, de la durée de l'enfermement, de son programme et des rapports entre intervenants et objets de l'enfermement un ensemble d'effets devant aboutir à la production d'une personne respectant des normes considérées comme essentielles à l'ordre social et sa reproduction.

C'est une dimension particulière du recours à l'enfermement pénal au tribunal des jeunes de Winnipeg, celle de la remise en situation sociojuridique d'enfance, que nous examinons. En effet, pour une proportion importante des jeunes à qui on a imposé une période de détention correctionnelle, il apparaît que l'intervention du tribunal résulte de leur refus de se conformer aux normes socio-juridiques les définissant comme des enfants ou des adolescents.

Il ne fait plus de doute, dans l'esprit de nombre de chercheurs (Chunn, 1992; Kett, 1977; Splane, 1965; Sutherland, 1976; Zeliser, 1985) depuis les travaux de P. Ariès (1973), que la représentation de l'enfance associée à l'époque moderne est le résultat d'une construction socio-historique. Les manifestations initiales, au Canada anglais, de cette construction remontent aux premières décennies du XIX $X^{\mathfrak{e}}$ siècle (Houston, 1975; Théoret, 1987). Comme le soulignent Rooke et Schnell (1983), cette construction s'est faite par l'inscription sociale dans les mentalités de quatre notions dominantes subsumant le cadre des représentations et des pratiques sociales concernant le groupe de personnes identifiées comme les jeunes: la dépendance, la ségrégation sociale, la protection et les responsabilités reportées.

Comment ces notions se sont-elles traduites dans le droit concernant les jeunes, ce droit qui définit et autorise la mise en marche du processus judiciaire réservé aux jeunes? Au Manitoba, la construction de l'enfance atteint sa forme moderne en 1924, alors que l'État, dans sa loi de protection des jeunes, se donne un rôle interventionniste dans la production des normes et des pratiques sociales à l'égard des jeunes ${ }^{3}$ et la capacité de faire

3. Le préambule de la loi manitobaine de 1922 sur la protection des jeunes (le Child Welfare Act) définit les objectifs impartis au Child Welfare Division, la partie de l'administration publique devant gérer les divers aspects de cette loi. Il est clair ici que 
respecter ces normes. Au plan juridique, quatre grandes notions normatives traversent le droit des jeunes et opèrent dans la décision de prise en charge judiciaire et, ultimement, dans la décision d'ordonner l'enfermement : le contrôle adulte acceptable, la présence d'un domicile fixe, l'interdiction de la présence des jeunes dans les lieux et les endroits publics sans être accompagnés d'un adulte responsable ou sans justifications admissibles et la moralisation du langage et des conduites, particulièrement au plan sexuel.

$\grave{A}$ cet égard, les normes juridiques que contient la loi manitobaine de protection des jeunes quant à ce que doit être la jeunesse constituent le substrat idéel de ce que doivent être les conditions de vie et de contrôle acceptables des jeunes, dont les jeunes dits délinquants. À l'exception de la définition des limites d'âge des jeunes tombant sous la juridiction des tribunaux pour jeunes, la Loi sur les jeunes délinquants adoptée en 1908 ne définissait pas ce qu'étaient les conditions de vie et de contrôle acceptables des jeunes. Elle ne remettait pas non plus en question le «quoi», le contenu du droit criminel canadien, mais plutôt son " comment», son application auprès des jeunes. Comme le souligne Trépanier (1991, pp. 207-208) dans son examen des discours législatifs précédant l'adoption, en 1908, de la Loi sur les jeunes délinquants, «[...], what was needed was an act stipulating how criminal law was to be applied to minors ${ }^{4}$ : before what type of court they would appear, what procedure would be followed and what measures would be used». De plus, les lois sur les jeunes délinquants et la protection des jeunes ont établi que les jeunes, à l'égard d'accusations similaires, peuvent tout autant être considérés comme délinquants que comme ayant besoin de protection. L'arrimage complet entre la gestion de la délinquance et celle de la protection, aux plans juridique, judiciaire, administratif et de l'intervention, brouille donc les distinctions auxquelles on pourrait s'attendre entre jeune délinquant et jeune ayant besoin de protection. Les mêmes tribunaux des jeunes délinquants doivent gérer tant des situations «simples» associées à des comportements relevant du droit criminel que des problématiques socio-juridiques «complexes" pour lesquelles la voie pénale a été choisie (Théorêt, 1993).

l'État assumera un rôle de leader ou de guide dans la manière dont les jeunes doivent être traités tant dans la famille que dans les institutions publiques. Les représentations et les attitudes sociales à l'égard des jeunes Manitobains sont donc transformées par cette loi, «through which the responsibility of the province rowards its child citizens may be more adequately recognized and discharged to the end that neglect of children may be minimized and a higher standard of child care incalculated and established thoughout the province».

4. C'est nous qui soulignons. 
Nous avançons donc que ce ne sont pas les tribunaux eux-mêmes qu'il faut qualifier selon des modèles de prise de décision, mais plutôt leur réaction ou leur fonctionnement à l'égard de situations-types et de types de déviance. Ainsi, on trouve le même tribunal fonctionnant selon une approche «de justice» (due process) lorsqu'il gère des situations «simples" associées à des comportements relevant du droit criminel et sans que les conditions de vie et de contrôle soient jugées problématiques, l'«approche criminelle», ou fonctionnant selon l'approche "traditionnelle " lorsqu'il doit gérer des problématiques socio-juridiques complexes relevant de l'état d'enfance et pour lesquelles la voie pénale a été choisie, l' "approche sociopénale". Ces deux types d'approche n'ont pas que des assises théoriques. Elles sont aussi fondées empiriquement, tant au plan juridique qu'à celui des pratiques judiciaires en ce qui a trait aux tribunaux canadiens. Voyons les différents éléments de ces approches.

L'approche criminelle est celle que nous aurons la plus de mal à conceptualiser. En effet, pour pouvoir penser cette approche, il nous faut remettre partiellement en question l'idée d'une séparation radicale, à laquelle nous avions ailleurs adhéré (Théorêt, 1987), entre justice pénale adulte et justice pénale des jeunes. On trouve bien les signes de cette séparation au plan légal, séparation dont les incidences se font sentir dans le nom que l'on donne aux jeunes ayant des démêlés avec la justice (les délinquants) et dans le type de procédures judiciaires minimales qui leur est réservé, au plan institutionnel, avec la création d'institutions judiciaires et correctionnelles qui sont réservées aux jeunes, et au plan idéel, dont la présence d'un discours causal spécifique têmoigne. Toutefois, la Loi sur les jeunes délinquants et son bras judiciaire, le tribunal des jeunes, n'en sont pas moins des institutions relevant du droit criminel. L'ensemble des normes comportementales codifiées dans ce droit devraient s'appliquer à toute la population canadienne en «âge de raisonner».

Il ne fait donc pas de doute que le tribunal des jeunes doit fonctionner formellement de façon légaliste, comme un tribunal criminel ordinaire ${ }^{5}$. Ceci se manifeste d'ailleurs par les obligations juridiques prévues dans la

5. Il nous semble particulièrement intéressant de constater que les seuls critères posant des limites formelles à l'activité sentencielle des juges des tribunaux des jeunes sont fondés sur l'âge : dans le cas des jeunes de moins de douze ans à l'encontre de qui if est illégal d'ordonner une détention dans un établissement correctionnel pour jeunes, et dans le cas des jeunes de moins de quinze ans, pour qui le défèrement vers les tribunaux ordinaires est impossible (Loi sur les jeunes délinquants (1929): art. 25 et $9(1))$. 
Loi sur les jeunes délinquants et, surtout, par les pratiques judiciaires observées au tribunal des jeunes de Winnipeg entre 1930 et 1959 :

- le juge de ce tribunal doit respecter des principes ordinaires de justice, s'assurer des faits reprochés aux jeunes et suivre les règles imposées par la procédure de juridiction sommaire prévue dans les cas d'infractions punissables sur déclaration sommaire de culpabilité (L.J.D., art. $5^{6}$ ). Cette procédure pourra être aussi informelle que possible, en autant qu'elle est compatible avec l'administration régulière de la justice ${ }^{7}$ (art. 17, 1929);

- lors d'accusations de vol ou de méfaits, la valeur des objets dérobés ou endommagés est toujours indiquée sur les dénonciations, témoignant de la prise en compte par le tribunal de considérations de "gravité des torts " causés aux victimes;

- l'emploi, dans un pourcentage élevé de causes, de la détention provisoire;

- bien que l'éventail sentenciel paraisse plus large que celui qui est normalement réservé aux adultes, on constate que l'amende, un type de peine que l'on peut difficilement qualifier de « réhabilitatrice ", occupe la première place parmi les sentences imposées aux jeunes ${ }^{8}$;

- le défèrement de causes vers les tribunaux ordinaires est utilisé à des fins stratégiques par le tribunal. Celui-ci permet au juge du tribunal des jeunes, se revêtant du titre de magistrat de tribunal ordinaire, d'ajouter la détention dans les institutions correctionnelles adultes à l'éventail sentenciel dont il dispose et, ainsi, d'augmenter la sévérité de la peine qu'il peut imposer à l'encontre de certains jeunes.

Donc, pour une part importante de ses activités, le tribunal des jeunes de Winnipeg fonctionne selon un modèle de justice criminelle ordinaire et

6. La Loi sur les jeunes délinquants à laquelle nous rêférons ci-après dans ce texte est la version adoptée en 1929. Notre étude portant sur des jeunes ayant compan après 1929. La loi de 1908 ayant été refondue et modifiée dans sa version de 1929, il s'agit de la version la plus appropriée dans le cadre de notre examen du rôle du droit dans les décisions sentencielles.

7. C'est nous qui soulignons.

8. Au tribunal des jeunes de Winnipeg, l'amende occupe une place privilégiée dans l'éventail sentenciel alors que, selon le réformateur W. L. Scott, rédacteur principal de la Loi sur les jeunes délinquants de 1908 et de plusieurs de ses amendements subséquents, «[...] it may be remarked that while, theoretically, a fine is out of place in the Juvenile Court, practical experience has indicated the usefulness of such a provision in certain exceptional cases " (Scott, 1930, p. 17). 
les sanctions imposées aux jeunes témoignent de finalités rétributivistes ou dissuasives ${ }^{9}$.

Bien évidemment, les tribunaux des jeunes, lorsqu'ils opèrent selon l'approche socio-pénale, reprennent une part importante du fonctionnement selon l'approche criminelle. Le caractère formaliste du tribunal est présent, mais le recours à la justice pénale vise à gérer des situations problématiques dont on ne retrouve pas l'équivalent légal dans le droit criminel applicable aux adultes. Quel type de déviance sociale peut être aussi important? Pourquoi recourir à la peine pour gérer des situations sociales?

Les situations reconstruites en accusations dans les dénonciations, telles que la fugue, l'école buissonnière, l'incorrigibilité, l'incontrôlabilité ou le fait d'avoir une conduite insatisfaisante sous la surveillance du tribunal, renvoient à une rupture à l'égard d'une ou de plusieurs des normes de ce que doit, légalement et socialement, être l'enfance. Le recours à la peine pour gérer une situation sociale devient donc plus compréhensible. D'une part, il s'agit là d'un des instruments dont disposent les législateurs pour parvenir à des fins de changement social, et cette possibilité a été prévue dans les lois de protection des jeunes. D'autre part, le refus de se conformer aux normes fondamentales du statut d'enfant est considéré comme une déviance importante appelant des mesures de redressement radicales pour la remise en situation d'enfance. En vertu du processus d'arrimage juridique et judiciaire, ce sont les tribunaux des jeunes qui doivent se charger de l'intervention auprès des jeunes dont la situation socio-juridique est irrégulière.

Voici les pratiques juridiques et judiciaires propres à l'approche sociopénale de fonctionnement au tribunal des jeunes de Winnipeg :

- un pouvoir discrétionnaire d'enquête sociale, permettant de s'assurer de la condition sociale et familiale des jeunes;

- l'obligation légale d'avoir des agents de probation parmi le personnel du tribunal, agents dont une des tâches est de procéder aux enquêtes sociales ;

- le peu d'importance que prend la mise en forme juridique des accusations portées contre les jeunes en vertu des délinquances dont les adultes ne peuvent être accusés. En plus de l'imprécision d'accusations comme celles d'être en dehors du contrôle de ses parents, d'être incorrigible, d'être incontrôlable, d'avoir une conduite insatisfaisante

9. La dimension exemplaire des sanctions est éliminée des considerations de sentence, car la publication du nom des jeunes, d'un rapport public sur le procès ou d'informations concernant les mesures prises par le tribunal à l'encontre d'un jeune est strictement interdite par la Loi sur les jeunes délinquants, 1929 (art. 12 (3)). 
sous surveillance du tribunal ou d'une société d'aide à l'enfance - accusations qui ne renvoient pas à des comportements ou à des actes précis - ces accusations sont utilisées de manière quasi interchangeable, indistinctement des événements amenant ou ramenant les jeunes devant le tribunal;

- le tribunal des jeunes est pro-actif dans la production de ses enquêtes. De plus, les informations recueillies lors de ces enquêtes sont utilisées pour la construction de l'identité délinquante des jeunes ;

- la détention dans les institutions correctionnelles, une mesure sévère et qui, dans le cas de la justice pénale des jeunes, devrait être une mesure de dernier recours, est largement imposée aux jeunes dont la situation correspond à la déviance à l'égard de leur statut socio-juridique.

L'analyse de la production sentencielle des tribunaux des jeunes ne peut faire l'économie d'un examen selon le sexe. Un nombre important d'études canadiennes et américiaines menées dans les années 1960 à 1980 démontrent clairement le traitement différentiel dont les filles font l'objet en vertu des lois de délinquance datant des premières décennies du $\mathrm{XX}^{\mathfrak{e}}$ siècle, tout particulièrement leur surreprésentation parmi les jeunes punis par la détention correctionnelle (Théorêt, 1991).

Le traitement différentiel selon le sexe n'est donc pas spécifique à ces deux dernières décennies. Il caractérise la pratique des tribunaux des jeunes depuis leur mise en place au début du $X X X^{\mathrm{e}}$ siècle. Il ne fait donc plus de doute maintenant que devant les tribunaux des jeunes, les filles et les garçons ne sont pas des « jeunes » à un même titre. Il existe des patrons de comportement différents et des attentes distinctes à l'égard des attitudes et des habitudes des jeunes selon leur sexe ${ }^{10}$ (Théorêt, 1987). Dans un tel

10. Étant donné l'intensité et la consistance des pratiques sentencielles différentielles a l'egard des filles, on pourrait être tenté de chercher dans les lois concernant les jeunes un fondement légal aux pratiques sexistes des tribunaux des jeunes. Ce n'est pas le cas des lois manitobaines concernant les jeunes. Toutefois, deux indices dans la loi canadienne sur les jeunes délinquants suggèrent un tel fondement juridique. En 1921, l'article 2 de la Loi sur les jeunes délinquants est amendé. Cette section porte sur la définition des termes et expressions utilisées dans la loi. L'alinéa portant sur la définition d' " enfant » est l'objet de la modification. À cette occasion, la loi signale que l'âge de la sujétion à la loi peut, à la demande de la province, par proclamation du gouverneur en conseil, être portée de moins de seize ans à moins de dix-huit ans. Cette décision est sujette à la discrétion des provinces. La notion légale d'enfant, sur la base de l'âge, est donc relative à la điscrétion des provinces. Mais plus encore, dans la même section, il est souligné que la discrétion des provinces de définir des personnes comme des «enfants " s'étend aussi au sexe des enfants : "Provided further that any such proclamation may apply to boys only or to girls only or to both boys and girls ». L'enfance ne 
contexte normatif genré, il existe un réel écart entre l' «enfant-fille» et l'«enfant-garçon " et entre la délinquance des filles et celle des garçons. De plus, tant les attentes à l'égard du résultat des sentences que leurs modalités d'application, et tout particulièrement le recours à la détention correctionnelle, ne sont pas les mêmes pour les jeunes, selon qu'il s'agisse de filles ou de garçons.

L'examen des décisions du tribunal des jeunes de Winnipeg selon le sexe, en tenant compte des approches criminelle et socio-pénale, permettra d'approfondir notre connaissance du fonctionnement des tribunaux des jeunes en regard du sexe des jeunes. Voyons maintenant comment nous comptons examiner les réactions sentencielles du tribunal des jeunes de Winnipeg à l'égard de ces deux types de situations.

\section{Remarques méthodologiques}

Les informations auxquelles nous recourons dans le cadre de cet article sont tirées des archives du tribunal des jeunes de Winnipeg ${ }^{11}$. Entre autres informations qui ont échappé à la destruction, on trouve 2570 dossiers $^{12}$ comprenant les documents relatifs aux divers contacts des jeunes avec le tribunal (documents judiciaires, policiers, correctionnels, sociaux, scolaires, médicaux et psychiatriques, entre autres) entre 1930 et 1959. Des données socio-démographiques et judiciaires ont été colligées en ce qui a trait au premier contact et au dernier contact des jeunes avec le tribunal. En d'autres termes, nous connaissons les informations sur les conditions de vie

se définirait plus seulement par le groupe d'âges auquel appartiennent les jeunes, mais aussi par leur sexe. En 1924, le Parlement canadien adopte une modification à la définition d'un jeune délinquant contenu dans la Loi sur les jeunes délinquants. On ajoute alors à la définition les mots «ou qui est coupable d'immoralité sexuelle ou de toute forme semblable de vice». Cette délinquance, qui n'a pas son équivalent chez les adultes, vise directement le comportement sexuel des filles (Théorêt, 1991).

11. Ces archives ont été identifiées et dépouillées par le professeur Len Kaminski et son équipe, du département de travail social de l'Université du Manitoba. Elies sont présentement conservées aux Public Archives of Manitoba à Winnipeg.

12. la période comprise entre 1930 et 1959,2570 dossiers judiciaires de jeunes ayant comparu pour des causes de "délinquance» devant le tribunal des jeunes de Winnipeg nous sont connus. Parmi ces 2570 jeunes ayant eu au moins un contact avec le tribunal, on trouve 2036 garçons $(79,2 \%)$ et 534 filles $(20,8 \%)$. De ce nombre, 2009 jeunes $(78,2 \%)$ se sont vu imposer des mesures sentencielles, $81,0 \%$ étant des garçons (1 627) et $19,0 \%$ des filles (382). Lors du dernier contact avec le tribunal, qui concerne 1332 jeunes $(51,8 \%)$, on trouve un pourcentage semblable de garçons et de filles à celui observé lors du premier contact ( $80,9 \%$ de garçons et $19,1 \%$ de filles). Les mesures sentencielles imposées par le tribunal nous sont connues dans $71,6 \%$ des cas (1 115 jeunes) et la proportion de filles $(19,3 \%)$ et de garçons $(80,7 \%)$ est maintenue. Le nombre des jeunes ayant fait l'objet de mesures sentencielles nous servira donc à déterminer la population. 
et le contrôle des jeunes et les décisions judiciaires du tribunal lorsque ces jeunes se sont présentés pour la première et la dernière fois devant le tribunal pour répondre à des accusations de délinquance.

La construction empirique de ces deux approches se fait en mettant en rapport la nature des accusations portées contre les jeunes et leurs conditions de vie et de contrôle ${ }^{13}$. Ainsi, dans l'approche criminelle, on trouve, d'une part, les délinquances dont la nature est traditionnellement associée à la sphère criminelle ou au maintien de l'ordre social : la délinquance contre les personnes et contre les biens, le vandalisme, les infractions concernant les véhicules moteurs, la délinquance associée aux armes à feu et aux drogues et la nuisance publique; d'autre part, la délinquance des jeunes dont les parents sont mariés et assument la garde légale et qui résident avec leurs parents au moment des contacts avec le tribunal, des jeunes dont les conditions de vie et de contrôle paraissent régulières.

Dans l'approche socio-pénale, on trouve, d'une part, des jeunes accusés exclusivement de types de délinquance dont des adultes ne peuvent être accusés, la plupart relevant des lois sociales, à l'exception de la clause d'immoralité sexuelle trouvée dans la définition d'un-e jeune délinquant-e (délinquance sexuelle ${ }^{14}$, fugue, absentéisme scolaire, incorrigibilité, absence

13. Au plan de l'analyse statistique, les approches criminelle et socio-pénale sont constituées comme des modèles purs, c'est-à-dire que chaque approche ne comprend que les dossiers des jeunes répondant totalement aux critères de construction précisés plus haut. Cette épuration des dossiers a entraîné une réduction du nombre de dossiers présents dans l'analyse. Ainsi, lors du premier contact, on trouve 1115 dossiers (sur 2009 dossiers ou $56,8 \%$ ) répondant à nos critères de constitution des approches criminelle et socio-pénałe. Ces dossiers se divisent entre 896 dossiers $(80,4 \%)$ répondant à l'approche criminelle et 219 dossiers $(19,6 \%)$ contenant l'ensemble des critères de l'approche socio-pénale. Lors du demier contact, 498 dossiers sur les 1115 dossiers $(44,7 \%)$ de la population ayant fait l'objet de mesures sentencielles et qui revient devant le tribunal répondent aux critères de sélection pour la constitution des approches criminelle et socio-pénale. Ceux-ci se divisent entre les approches criminelle (340 dossiers ou $68,3 \%$ ) et socio-pénale ( 158 dossiers ou $31,7 \%$ ). On constate qu'un pourcentage plus élevé de dossiers correspondant à l'approche socio-pénale contient des contacts multiples avec le tribunal (158 dossiers sur 219 ou $71,1 \%$ ). Dans le cas des jeunes dont les dossiers témoignent de l'appartenance à l'approche criminelle de fonctionnement, les contacts multiples concernent $37,9 \%$ des dossiers (340 dossiers sur 896).

14. On pourrait penser que la délinquance sexuelle, dans le cas des garçons, ne devrait pas être utilisée comme variable discriminante dans la constitution des approches criminelle et socio-pénale. Pourtant, l'examen des dossiers nous a permis de constater que les accusations de délinquance sexuelle portées contre les garçons sont pour la grande majorité liées au fait d'avoir eu des rapports sexuels mutuellement consentis avec des filles ou des rapports homosexuels, la plupart du temps avec des adultes. Les accusations d'agressions sexuelles sont rares dans les dossiers. 
d'accusation précise ${ }^{15}$, etc.), ainsi que la délinquance relevant des contacts avec le tribunal ou les institutions correctionnelles (unsatisfactory under the supervision of the Court, under the supervision of the superintendent of the Manitoba Home for Boys, for Girls ou no charge laid). D'autre part, les jeunes dont les conditions de vie et de contrôle sont caractérisées par l'ensemble des situations problématiques suivantes: situations parentale (parents séparés, divorcés ou décédés, mère célibataire), légale (pupille du tribunal ou du directeur du Child Welfare Department) et résidentielle (ne résidant pas avec leurs parents ou des parents proches) irrégulières.

Nous nous proposons ici d'examiner les décisions sentencielles du tribunal des jeunes de Winnipeg entre 1930 et 1959 en recourant à ces deux approches de fonctionnement du tribunal. La présence de ces deux approches suggère que la compréhension des décisions sentencielles des tribunaux des jeunes ainsi que des écarts que l'on retrouve se comprend mieux lorsque l'on accepte la diversité des situations gérées par ces tribunaux. C'est d'ailleurs dans ce cadre que les décisions portant sur la mesure la plus sévère que puissent imposer ces tribunaux, la détention correctionnelle, puissent le mieux être mises en lumière.

Enfin, on constate, en comparaison de Ja population totale, qu'en vertu des caractéristiques de proportionnalité concernant la représentation selon le sexe lors des contacts uniques et des contacts multiples, la sélection des dossiers, selon qu'ils correspondent aux caractérisiques des approches criminelle ou socio-pénale, n'induit pas de biais perceptibles dans cette représentation ${ }^{16}$.

15. On trouve dans les dossiers des jeunes des dénonciations (Information and Complaint) sur lesquelles a été tamponnée l'inscription No charge Laid. Il s'agit pour la plupart de jeunes déjà sous la surveillance du tribunal et dont le tribunal peut obtenir qu'ils comparaissent sans qu'une accusation soit portée contre eux, en vertu de l'article 20 (3) de la Loi sur les jeunes délinquants.

16. Au plan de la division selon le sexe, des dossiers compris dans les approches criminelle et socio-pénale au premier contact, on trouve une représentation très similaire à celle de la population ayant fait l'objet de mesures sentencielles. En effet, alors qu'au premier contact on comptait $19,0 \%$ de filles et $81,0 \%$ de garçons dans la population totale, on trouve $18,2 \%$ de filles et $81,8 \%$ de garçons dans les dossiers appartenant aux approches criminelle et socio-pénale. Dans ces approches, la distribution de la population selon le sexe témoigne d'une différence importante : au premier contact, les 896 dossiers répondant à l'approche criminelle se divisent très inégalement entre les dossiers de garçons $(92,1 \%$ ou 825 dossiers) et les dossiers de filles $(7,9 \%$ ou 71 dossiers). Les 219 dossiers correspondant à l'approche socio-pénale montrent clairement une surreprésentation des filles dans cette approche : les dossiers de garçons constituent $39,7 \%$ (ou 87 dossiers) de la population répondant à cette approche, ceux des filles en représentant $60,3 \%$ (ou 132 dossiers). 


\section{Approches criminelle et socio-pénale, sexe et décisions sentencielles}

Voyons maintenant le résultat de l'examen des décisions sentencielles du tribunal des jeunes de Winnipeg, en tenant compte tant du sexe des jeunes qui devaient se présenter devant ce tribunal que des approches de fonctionnement criminel et socio-pénal que nous avons construites. Pour ce faire, nous présenterons les résultats lors des premier et dernier contacts des jeunes avec le tribunal. Les tableaux-synthèses qui suivent présentent un certain nombre de variables socio-démographiques et judiciaires mises en relation avec nos approches des tribunaux et le sexe des jeunes pris en charge par le tribunal des jeunes de Winnipeg lors des premier et dernier contacts.

\section{Au premier contact}

L'âge des jeunes lors du premier contact nous permet déjà d'apprécier les différences entre les jeunes selon que leur dossier correspond aux approches criminelle ou socio-pénale (tableau 1). Ainsi, les jeunes dont les dossiers répondent aux caractéristiques de l'approche socio-pénale sont un peu plus âgés que ceux dont le premier contact correspond à l'approche criminelle. On constate cependant qu'indépendamment de l'approche de fonctionnement, les filles sont plus âgées que les garçons. Il s'agit d'une différence qui est accentuée dans le cas des filles dont le dossier correspond à l'approche socio-pénale (+ 0,9 année et + 1,3 année).

Les différences de fonctionnement du tribunal de Winnipeg selon les approches sont importantes lorsque l'on considère les décisions de mettre sous garde les jeunes lors de leur procès. Ainsi, le cinquième des jeunes dont le dossier correspond à l'approche criminelle seront détenus durant une partie ou l'entièreté de leus procès (filles : 20,5\%; garçons : 18,5\%), alors que ce sera le cas de plus de la moitié des jeunes dont les dossiers correspondent à l'approche socio-pénale (filles : $63,4 \%$; garçons : $52,5 \%$ ). On constate toutefois que les filles dont le dossier répond aux caractéristiques

Lors du dernier contact, la distribution selon le sexe des jeunes n'est pas modifiée de manière importante. Ainsi, on trouvait $19,0 \%$ de filles et $81,0 \%$ de garçons dans la population ayant fait l'objet de mesures sentencielles. Dans les dossiers compris dans les approches criminelle et socio-pénale, on compte $22,3 \%$ de filles et $77,7 \%$ de garçons. Les différences trouvées, lors du premier contact, dans la répartition selon le sexe dans les approches criminelle et socio-pénale se maintiennent lors du demier contact avec le tribunal. Les dossiers correspondant à l'approche criminelle sont constitués, dans un pourcentage de $95,6 \%$ (340 dossiers) de dossiers de garçons, et dans un pourcentage de $4,4 \%$ ( 15 dossiers), de dossiers de filles. Les dossiers compris dans l'approche socio-pénale sont répartis, selon un pourcentage de $39,2 \%$ et $60,8 \%$, entre les dossiers de garçons ( 62 dossiers) et les dossiers de filles ( 96 dossiers). 
de l'approche socio-pénale sont, proportionnellement, plus frappées par la mesure de mise sous garde. Le nombre moyen de comparutions lors du premier contact, selon que le dossier des jeunes correspond à une approche ou à l'autre, montre aussi la différence de fonctionnement du tribunal : alors que les jeunes dont le dossier répond aux caractéristiques de l'approche criminelle auront comparu en moyenne 1,8 fois devant le tribunal, ceux dont le dossier correspond à l'approche socio-pénale se seront présentés en moyenne 2,4 fois.

Malgré des différences entre les pourcentages, les filles et les garçons dont le dossier correspond à l'approche criminelle sont accusés des mêmes types d'infraction, la délinquance contre les biens comprenant la majorité des types d'accusation (filles: 79,5\%; garçons: $66,3 \%$ ), alors que les accusations de nuisance publique (filles : $8,4 \%$; garçons : $15,4 \%$ ) et celles liées aux véhicules moteurs (filles : $6,0 \%$; garçons : $7,3 \%$ ) constituent les autres catégories d'accusation principales. Les jeunes dont le dossier répond aux caractéristiques de l'approche socio-pénale sont surtout accusés en vertu d'infractions spécifiques aux jeunes (filles: $59,7 \%$; garçons: $48,2 \%$ ). Des différences existent toutefois: on trouve un pourcentage plus élevé de filles $(28,1 \%)$ faisant l'objet d'accusation d'immoralité sexuelle que de garçons $(6,0 \%)$. En revanche, les garçons sont plus souvent amenés devant le tribunal sans qu'une accusation précise soit portée contre eux $(45,8 \%)$ que les filles $(12,2 \%)$.

Au plan des sentences ${ }^{17}$ imposées, on constate ici aussi l'impact des approches sur le mode de fonctionnement du tribunal des jeunes de Winnipeg. À chacune de ces approches correspondent des modes de punition particuliers. Ainsi, les punitions procentuellement les plus importantes imposées aux jeunes dont le dossier correspond à l'approche criminelle sont l'amende (filles: $32,4 \%$, garçons : $40,1 \%$ ), la probation (filles : $40,8 \%$; garçons : $33,9 \%$ ), la réprimande (filles : $26,9 \%$; garçons : $34,1 \%$ ) et la restitution (filles : $29,6 \%$; garçons : $29,8 \%$ ). Les jeunes dont le dossier correspond à l'approche socio-pénale seront surtout punis par la détention correctionnelle (filles : $43,9 \%$; garçons : $47,1 \%$ ), la probation (filles : $40,9 \%$; garçons : $23,0 \%$ ) et, marginalement, l'amende (filles : $3,8 \%$; garçons : $11,5 \%$ ). Une

17. Pour ne pas perdre la diversité et la multiplicité des sentences lors d'un même contact, nous recourons à une procédure désignée sous le nom anglais de multiple responses. Les tableaux produits à l'aide de cette procédure de traitement statistique ne permettent pas de connaître le nombre total de décisions ou de sentences prises lors d'un contact dans un dossier, mais plutôt la présence ou non des divers types de sentences lors de ce contact. Ainsi, notre analyse des sentences se présente sous la forme de pourcentages calculés sur le total des différentes sentences trouvées dans les dossiers lors des premier et demier contacts plutôt que sous la forme de la sentence la plus importante dans chaque dossier. 
partie significative des jeunes appartenant à cette dernière approche font l'objet d'une libération (filles: 12,1\%; garçons : 17,2\%).

Tableau 1

Synthèse statistique de certaines pratiques judiciaires et sentencielles du tribunal des jeunes de Winnipeg lors du premier contact

\begin{tabular}{|c|c|c|c|c|}
\hline \multirow[b]{3}{*}{ Sexe } & \multicolumn{4}{|c|}{ PREMIER CONTACT ( 1115 dossiers $)$} \\
\hline & \multicolumn{2}{|c|}{$\begin{array}{l}\text { APPROCHE CRIMINELLE } \\
(896 ; 80,4 \%)\end{array}$} & \multicolumn{2}{|c|}{$\begin{array}{c}\text { APPROCHE SOCIO-PÉNALE } \\
(219 ; 19.6 \%) \\
\end{array}$} \\
\hline & $\begin{array}{l}\text { Garçons : } 825 \\
(92,1 \%)\end{array}$ & $\begin{array}{l}\text { Filles : } 71 \\
(7,9 \%)\end{array}$ & $\begin{array}{c}\text { Garçons : } 87 \\
(39,7 \%)\end{array}$ & $\begin{array}{l}\text { Filles : } 132 \\
(60,3 \%)\end{array}$ \\
\hline Âge moyen & 13,7 & 14,6 & 13,9 & 15,2 \\
\hline $\begin{array}{l}\text { Détention } \\
\text { provisoire }\end{array}$ & $18,5 \%$ & $20,5 \%$ & $52,5 \%$ & $63,4 \%$ \\
\hline $\begin{array}{l}\text { Nombre } \\
\text { moyen de } \\
\text { comparutions }\end{array}$ & 1,8 & 1,8 & 2,4 & 2,4 \\
\hline $\begin{array}{l}\text { Nature des } \\
\text { accusations }\end{array}$ & $\begin{array}{l}\text { Biens }^{18}: 66,3 \% \\
\text { Nuisance : } 15,4 \% \\
\text { Véhicules : } 7,3 \%\end{array}$ & $\begin{array}{l}\text { Biens }^{19}: 79,5 \% \\
\text { Nuisance : } 8,4 \% \\
\text { Véhicules : } 6,0 \%\end{array}$ & $\begin{array}{l}\text { Spécifique : } 48,2 \% \\
\text { Aucune : } 45,8 \% \\
\text { Sexuelle : } 6,0 \%\end{array}$ & $\begin{array}{l}\text { Spécifique : } 59,7 \% \\
\text { Sexuelle : } 28,1 \% \\
\text { Aucune : } 12,2 \%\end{array}$ \\
\hline $\begin{array}{l}\text { Nature des } \\
\text { sentences }\end{array}$ & $\begin{array}{l}\text { Amende } 20: 40,1 \% \\
\text { Réprimande : } \\
34,1 \% \\
\text { Probation : } 33,9 \% \\
\text { Restitution: } \\
29,8 \%\end{array}$ & $\begin{array}{l}\text { Probation }^{21}: \\
40,8 \% \\
\text { Amende : } 32,4 \% \\
\text { Restitution : } \\
29,6 \% \\
\text { Réprimande : } \\
26,8 \%\end{array}$ & $\begin{array}{l}\text { Détention } 22: \\
47,1 \% \\
\text { Probation : } 23,0 \% \\
\text { Libération : } 17,2 \% \\
\text { Amende : } 11,5 \%\end{array}$ & $\begin{array}{l}\text { Détention } 23 \text { : } \\
43,9 \% \\
\text { Probation : } 40,9 \% \\
\text { Libération : } 12,1 \% \\
\text { Amende : } 3,8 \%\end{array}$ \\
\hline
\end{tabular}

18. Les pourcentages à l'égard des autres types d'accusation sont : vandalisme: $5,5 \%$; infractions contre la personne : $3,0 \%$; armes : $1,9 \%$; drogues : $0,7 \%$.

19. Les pourcentages à l'égard des autres types d'accusation sont: infractions contre la personne : $3,6 \%$; vandalisme : $2,4 \%$.

20. Les pourcentages concernant les autres sentences sont les suivants : libération : $11,9 \%$; divers : $9,1 \%$; punition corporelle : $2,8 \%$; détention correctionnelle : $2,5 \%$; placement en foyer d'accueil : $0,5 \%$; institutionnalisation psychiatrique : $0,1 \%$.

21. Les pourcentages concernant les autres sentences sont les suivants: libération : $16,9 \%$; divers : $7,0 \%$; détention correctionnelle : $2,8 \%$; placement en foyer d'accueil : $1,4 \%$.

22. Les pourcentages concernant les autres sentences sont les suivants : réprimande : $6,9 \%$; restitution : $2,3 \%$; punition corporelle : $1,1 \%$.

23. Les pourcentages concernant les autres sentences sont les suivants: réprimande : $0,8 \%$; placement en foyer d'accueil : $0,8 \%$; divers : $0,8 \%$. 


\section{Au dernier contact}

Les pratiques judiciaires lors du dernier contact des jeunes avec le tribunal ne présentent plus des différences aussi importantes que lors du premier contact (tableau 2). Ainsi, un pourcentage plus important de jeunes dont le dossier correspond à l'approche criminelle sont mis sous garde (filles : $33,3 \%$; garçons : $32,6 \%$ ) alors que, même s'il demeure supérieur, le pourcentage n'augmente pas pour les jeunes dont le dossier répond aux critères de l'approche socio-pénale (filles: $48,0 \%$; garçons: $54,1 \%$ ). De même, le nombre moyen de comparutions lors du dernier contact a augmenté pour les jeunes dont le dossier correspond à l'approche criminelle (filles : 2,4 comparutions; garçons : 2,2 comparutions) alors qu'il est resté stable dans le cas des jeunes dont le dossier répond aux critères de l'approche socio-pénale (filles : 2,4 comparutions; garçons : 2,6 comparutions).

Au plan des types d'accusation qui sont portées contre les jeunes lors du dernier contact, peu de différences avec les accusations portées lors du premier contact sont notables dans les dossiers des jeunes correspondant à l'approche criminelle. Les mêmes types d'accusations reviennent, et ce dans des pourcentages semblables: délinquance contre les biens (filles: $66,7 \%$; garçons: $63,5 \%$ ), nuisance publique (filles: $5,6 \%$; garçons: $10,0 \%)$. On constate toutefois que les filles sont, en second lieu, accusées de vandalisme $(22,2 \%)$ alors que pour les garçons, les accusations liées aux véhicules moteurs sont plus importantes en termes de pourcentage que les accusations de nuisance publique $(14,1 \%)$. Les jeunes dont les dossiers correspondent à l'approche socio-pénale lors du dernier contact sont l'objet des mêmes types d'accusation que lors du premier contact. Toutefois, la distribution en pourcentage entre les types d'accusation s'est modifiée. Ainsi, la délinquance spécifique aux jeunes prend une plus grande place dans les accusations (filles : $82,7 \%$; garçons : $71,3 \%$ ) alors que les pourcentages d'accusation de délinquance sexuelle (filles: 12,0\%; garçons: $7,0 \%$ ) et l'absence d'accusation précise (filles : 5,3\%; garçons: $21,7 \%$ ) sont réduits respectivement pour les filles et les garçons.

Les types de sentences qui sont imposées par le tribunal aux jeunes dont le dossier correspond à l'approche criminelle ne montrent pas de signes d'une intensification de la punitivité. On constate cependant une différence selon le sexe dans les pourcentages d'imposition: les filles sont surtout punies par des périodes de probation $(60,0 \%)$, par la restitution $(40,0 \%$; garçons : $22,8 \%)$ et par la détention correctionnelle $(20,0 \%)$, alors que les garçons se voient imposer des amendes $(42,2 \%)$ et des périodes de probation $(38,5 \%)$. La réprimande occupe la quatrième place en termes de pourcentage, tant pour les filles $(13,3 \%)$ que pour les garçons $(20,3 \%)$, parmi les sentences imposées aux jeunes dont le dossier correspond à l'approche criminelle. Les principaux types de sentence imposés aux jeunes dont le dossier rêpond aux caractéristiques de l'approche socio- 
pénale ne changent pas entre le premier et le dernier contact. On constate cependant un recours plus important à la détention correctionnelle que lors de la décision sentencielle au premier contact, et ce tant pour les filles $(60,5 \%)$ que pour les garçons $(59,7 \%)$. L'imposition de périodes de probation (filles: 17,7\%; garçons : $11,3 \%$ ) et la libération (filles : $14,6 \%$; garçons: $12,9 \%$ ) se retrouve de même dans l'éventail des principales sentences imposés par le tribunal. L'amende est toutefois plus souvent imposée aux garçons $(21,0 \%)$ qu'aux filles $(4,2 \%)$.

Tableau 2

Synthèse statistique de cersaines pratiques judiciaires du iribunal des jeunes de Winnipeg lors du dernier contact

\begin{tabular}{|c|c|c|c|c|}
\hline \multirow[b]{3}{*}{ Sexe } & \multicolumn{4}{|c|}{ DERNIER CONTACT (498 dossiers ou $44,7 \%$ ) } \\
\hline & \multicolumn{2}{|c|}{$\begin{array}{c}\text { APPROCHE CRIMINELLE } \\
(340 ; 68,3 \%)\end{array}$} & \multicolumn{2}{|c|}{$\begin{array}{c}\text { APPROCHESOCIO-PÉNALE } \\
(158 ; 31,7 \%)\end{array}$} \\
\hline & $\begin{array}{c}\text { Garçons : } 325 \\
(95,6 \%)\end{array}$ & $\begin{array}{l}\text { Filles : } 15 \\
(4,4 \%)\end{array}$ & $\begin{array}{c}\text { Garçons :62 } \\
(39,2 \%)\end{array}$ & $\begin{array}{l}\text { Filles : } 96 \\
(60,8 \%)\end{array}$ \\
\hline $\begin{array}{l}\text { Détention } \\
\text { provisoire }\end{array}$ & $32,6 \%$ & $33,3 \%$ & $54,1 \%$ & $48,0 \%$ \\
\hline $\begin{array}{l}\text { Nombre } \\
\text { moyen de } \\
\text { comparutions }\end{array}$ & 2,2 & 2,4 & 2,6 & 2,4 \\
\hline $\begin{array}{l}\text { Nature des } \\
\text { accusations }\end{array}$ & $\begin{array}{l}\text { Biens }^{24}: 63,5 \% \\
\text { Véhicules : } 14,1 \% \\
\text { Nuisance : } 10,0 \%\end{array}$ & $\begin{array}{l}\text { Biens }^{25}: 66,7 \% \\
\text { Vandalisme: } \\
22,2 \% \\
\text { Nuisance : } 5,6 \%\end{array}$ & $\begin{array}{l}\text { Spécifique : } 71,3 \% \\
\text { Aucune : } 21,7 \% \\
\text { Sexuelie : } 7,0 \%\end{array}$ & $\begin{array}{l}\text { Spécifique : } 82,7 \% \\
\text { Sexuelle : } 12,0 \% \\
\text { Aucune : } 5,3 \%\end{array}$ \\
\hline $\begin{array}{l}\text { Nature des } \\
\text { sentences }\end{array}$ & $\begin{array}{l}\text { Amende } 26: 42,2 \% \\
\text { Probation : } 38,5 \% \\
\text { Restitution : } 22,8 \% \\
\text { Réprimande : } \\
20,3 \%\end{array}$ & $\begin{array}{l}\text { Probation } 27: \\
60,0 \% \\
\text { Restitution : } 40,0 \% \\
\text { Détention : } 20,0 \% \\
\text { Réprimande : } \\
13,3 \%\end{array}$ & $\begin{array}{l}\text { Détention } 28: \\
59,7 \% \\
\text { Amende : } 21,0 \% \\
\text { Libération : } 12,9 \% \\
\text { Probation : } 11,3 \%\end{array}$ & $\begin{array}{l}\text { Détention } 29: \\
60,5 \% \\
\text { Probation : } 17,7 \% \\
\text { Libération : } 14,6 \% \\
\text { Amende : } 4,2 \%\end{array}$ \\
\hline
\end{tabular}

24. Les pourcentages à l'égard des autres types d'accusation sont : vandalisme : $4,9 \%$; infractions contre la personne : $4,4 \%$; armes : $2,4 \%$; drogues : $0,7 \%$.

25. Les pourcentages a l'égard des autres types d'accusation sont : vêhicules moteurs : $5,6 \%$.

26. Les pourcentages concernant les autres sentences sont les suivants : détention correctionnelle : $11,4 \%$; liberation : $10,5 \%$; divers : $9,2 \%$; punition corporelle : $0,6 \%$.

27. Les pourcentages concemant les autres sentences sont les suivants: liberation : $13,3 \%$; amende : $6,7 \%$; divers : $6,7 \%$.

28. Les pourcentages concernant les autres sentences sont les suivants : restitution : $3,2 \%$; punition corporelle : $1,6 \%$.

29. Les pourcentages concernant les autres sentences sont les suivants: divers : $2,1 \%$; institutionnalisation psychiatrique : $2,1 \%$; réprimande : $1,0 \%$; restitution : $1,0 \%$. 


\section{CONCLUSION}

Il nous semble donc important, pour comprendre les décisions sentencielles du tribunal des jeunes de Winnipeg et plus particulièrement les décisions de détention correctionnelle, de tenir compte de ce que sont les tribunaux des jeunes avant les modifications législatives qui font passer les jeunes ayant des démêlés avec la justice pénale du statut de "délinquants » à celui de «contrevenants". Plus particulièrement, il nous paraît essentiel de ne pas réduire le rôle des tribunaux des jeunes à des organismes fonctionnant selon un seul mode et dont les décisions sentencielles relèveraient de cet unique mode de fonctionnement, que celui-ci soit dit «traditionnel » ou de due process ou encore influencé par des considérations ou légales ou sociales.

Les tribunaux des jeunes, au Canada à tout le moins, ont été mis en place dans le cadre d'un arrimage entre le droit social des jeunes et criminel. Le recours aux sentences les plus sévères, l'enfermement tout particulièrement, pour imposer le respect des normes de l'enfance est tout à fait dans l'esprit de ces droits. De façon récursive, ces droits consacrent la transformation sociale de la représentation de l'enfance, alors que les pratiques judiciaires pénales qui découlent de leur application, particulièrement les sentences les plus importantes, cautionnent la nécessité de normes différentes à l'égard des jeunes dans un droit qui leur est particulier. Il existe donc une situation que les législateurs ont certes prévue mais qui n'en laisse pas moins les intervenants du tribunal des jeunes perplexes: des jeunes, dont les conditions de vie et de contrôle sont perçues comme appelant une intervention sociale refusent cette intervention et décident de prendre en mains leur propre destinée. Ces jeunes se soustraient à la surveillance adulte, fréquentent les lieux et les endroits publics, résident là où bon leur semble et décident de leur propre moralité sexuelle. La délinquance de ces jeunes à l'égard du statut social d'enfant entraîne une réaction de type pénal du tribunal des jeunes. Il s'agit d'une situation numériquement importante dans le cadre du fonctionnement du tribunal des jeunes de Winnipeg. Lors du premier contact, plus du quart $(19,6 \%)$ des jeunes de notre sous-population qui se présentent devant le tribunal s'y retrouvent pour cette raison. Lors du dernier contact, c'est le cas de près du tiers $(31,6 \%)$ des jeunes.

Il ne faut toutefois pas croire que cet arrimage équivaut à une assimilation complète entre les pratiques pénales liées à l'application du droit criminel et celles relevant du droit social des jeunes. Au contraire, le tribunal des jeunes de Winnipeg réagit de façon différente à l'égard de ces deux situations-types de délinquance. Lors du premier contact avec le tribunal, les pratiques judiciaires montrent tant la gravité perçue de la situation correspondant à l'approche socio-pénale, comme en témoignent les forts 
pourcentages de mise sous garde, que la difficulté à définir la situation et à décider des mesures à prendre, comme le montre le nombre de comparutions nécessaires à l'issue du procès. Les sentences imposées sont aussi significatives et renvoient au statut d'enfants des jeunes dans plus des trois quarts des dossiers : la détention correctionnelle et la probation permettent de rétablir la surveillance adulte et la fixité domiciliaire, de réduire ou de contrôler la présence des jeunes dans les lieux et les endroits publics et de réaffirmer certaines valeurs relatives à la morale sexuelle. À cet égard, l'enfermement pénal des jeunes est une suite logique du processus d'exclusion sociale des jeunes.

La réaction du tribunal de Winnipeg à l'égard des jeunes dont le dossier correspond à l'approche criminelle paraît plus proche de ce que l'on attend des tribunaux pénaux pour adultes tout en conservant une spécificité liée au droit des jeunes. L'imposition d'amendes dans des pourcentages importants, une mesure qui a priori n'apparaît pas comme particulièrement liée au statut d'enfant, doit être mise en rapport avec la perception que le tribunal se fait de la nature de la délinquance et de la meilleure façon d'y réagir. Il s'agit d'une délinquance "normale», "attendue", de même nature que celle commise par les adultes. On constate cependant que le tribunal utilise souvent la restitution, une mesure expressément prévue dans la Loi sur les jeunes délinquants et qui n'a pas son équivalent dans l'éventail des sentences réservées aux adultes.

Lors du dernier contact avec le tribunal, les différences au plan des pratiques judiciaires entre les jeunes dont le dossier correspond à l'une ou l'autre approches se sont amenuisées. Dans un cas comme dans l'autre, il s'agit de jeunes ayant eu des contacts multiples avec le tribunal, la répétition de ces contacts manifestant l'échec relatif des mesures prises précédemment. Toutefois, ceci semble être le cas surtout des jeunes dont le dossier relève de l'approche criminelle. En effet, alors que les pourcentages de mise sous garde et le nombre moyen de comparutions des jeunes dont le dossier correspond à l'approche socio-pénale sont marqués par une baisse. particulièrement chez les filles, les jeunes dont le dossier relève de l'approche criminelle sont en termes de pourcentage plus souvent mis sous garde et leur procès exige un nombre plus élevé de comparutions que lors de leur premier contact avec le tribunal. Jusqu'à un certain point, les caractéristiques judiciaires de ces jeunes se rapprochent de celles des jeunes dont le dossier correspond à l'approche socio-pénale. Cependant, les ressemblances s'arrêtent ici. Les types de sentences imposées aux jeunes, selon qu'ils appartiennent à une approche où l'autre correspondent presque exactement au mêrne patron que lors du premier contact. Ainsi, au plan des décisions sentencielles, le tribunal des jeunes de Winnipeg fait montre d'une constance sans faille à l'égard de la punition des jeunes, qu'il 
s'agisse des jeunes dont le dossier correspond à l'approche criminelle ou de ceux dont le dossier relève de l'approche socio-pénale.

Il est surprenant, compte tenu des études précédentes, que cette constance en vertu des approches de fonctionnement se trouve aussi lorsqu'examiné en fonction du sexe. Toutefois, force nous est de conclure que l'approche criminelle correspond à un mode de fonctionnement du tribunal s'adressant presque exclusivement aux garçons, alors que l'approche sociopénale correspond, dans des pourcentages assez égaux, à un mode de fonctjonnement s'adressant tant aux filles qu'aux garçons. Malgré des différences importantes entre le nombre de filles et de garçons dont le dossier correspond à l'approche criminelle, les types de sentences pour ces deux groupes sont similaires. Cette similarité des types de sentences selon le sexe est encore plus grande pour les jeunes dont le dossier relève de l'approche socio-pénale.

On constate cependant, en examinant les pourcentages des types de sentence, que l'amende est plus largement imposée aux garçons, indépendamment de l'approche à laquelle leur dossier correspond alors que les mesures de surveillance (probation) sont ordonnées plus souvent dans le cas des filles. En dépit de l'absence apparente de traitement différentiel généralisé, l'utilisation de certaines mesures sentencielles démontre qu'à l'égard des garçons, le tribunal des jeunes de Winnipeg opère d'une façon plus rétributiviste et dissuasive, alors qu'à l'égard des filles, les objectifs de surveillance et de contrôle liés au statut d'enfant prennent une place plus importante, même dans l'approche criminelle. 


\section{BIBLIOGRAPHIE}

ARIÈS, Ph. (1973), L'Enfant et la vie familiale sous l'ancien régime, Paris, Seuil.

CHUNN, D. (1992), From Punishment to Doing Good: Family Courts and Socialized Justice in Ontario, 1880-1940, Toronto, University of Toronto Press.

HOUSTON, S. F. (1975), "Victorian Origins of Juvenile Delinquency: a Canadian Experience », in M. B. Katz and P. H. Mattingly (éd.) Education and Social Change. Themes from Ontario's Past, New York, New York University Press, pp. 129-142.

KETT, J. F. (1977), Rites of Passage. Adolescence in America, 1790 to the Present, New York, Basic Books.

SPLANE, R. B. (1965), Social Welfare in Ontario, (1791-1893), Toronto, University of Toronto Press.

SUTHERLAND, N. (1976), Children in English Canadian Society : Framing the Twentieth Century Consensus, Toronto, University of Toronto Press

ROOKE, P. T, SCHNELL, R. L. (1983), Discarding the Asylum: From Child Rescue to the Welfare State in English-Canada (1800-1950), Lanham Md., University Press of America.

SCOTT, W. L. (1930), The Juvenile Court in Law and The Juvenile Court in Action. Ottawa, Canadian Council on Child Welfare (publication $\pi^{\circ} 34$ ).

THÉORÊT, B. (1993), Espace social et régulation juridique des jeunes. Le Fonctionnement du tribunal des jeunes de Winnipeg, Université du Québec à Montréal, Montréal, Département de sociologie, thèse de doctorat.

THÉORÊT, B. (1991), «Régulation juridique pénale des mineur-es et discrimination à l'égard des filles: la clause de 1924 amendant la Loi sur les jeunes délinquants ", Revue Femmes et Droit, vol. 4, n 2, pp. 539-555.

THÉORÊT, B. (1987), L'Histoire du contrôle social et pénal des filles au Canada anglais de 1800 a 1930, Université de Montréal, École de criminologie, mémoire de maîtrise.

TRÉPANIER, J. (1991), « The Origins of the Juvenile Delinquents Act of 1908 : Controlling Delinquency Through Seeking its Causes and Through Youth Protection », in Russell Smandych, Gordon Dodds et Alvin Esau (éd.) Dimensions of Childhood. Essays on the History of Children and Youth in Canada. Winnipeg, Legal Research Institute of the University of Manitoba, pp. 205-232.

ZELISER. V. (1985), Pricing the Priceless Child: The Changing Social Value of Children, New York, Basis Books. 